\title{
ON THE GEOMETRY OF FIXED POINTS FOR SELF-MAPPINGS ON $S$-METRIC SPACES
}

\author{
Nihal TAŞ and Nihal ÖZGÜR
}

Balıkesir University, Department of Mathematics, 10145, Balıkesir, TURKEY

\begin{abstract}
In this paper, we focus on some geometric properties related to the set Fix $(T)$, the set of all fixed points of a mapping $T: X \rightarrow X$, on an $S$-metric space $(X, \mathcal{S})$. For this purpose, we present the notions of an $S$-Pata type $x_{0^{-}}$ mapping and an $S$-Pata Zamfirescu type $x_{0}$-mapping. Using these notions, we propose new solutions to the fixed circle (resp. fixed disc) problem. Also, we give some illustrative examples of our main results.
\end{abstract}

\section{Introduction and Preliminaries}

The notion of an $S$-metric space was introduced as a generalization of a metric space as follows:

Definition 1. [20] Let $X$ be a nonempty set and $\mathcal{S}: X^{3} \rightarrow[0, \infty)$ be a function satisfying the following conditions for all $x, y, z, a \in X$ :

(1) $\mathcal{S}(x, y, z)=0$ if and only if $x=y=z$,

(2) $\mathcal{S}(x, y, z) \leq \mathcal{S}(x, x, a)+\mathcal{S}(y, y, a)+\mathcal{S}(z, z, a)$.

Then $S$ is called an $S$-metric on $X$ and the pair $(X, \mathcal{S})$ is called an $S$-metric space.

Many researchers have studied on $S$-metric spaces to obtain new fixed point results and some applications (see $7,9,10,15,21$ and the references therein). Also, the relationship between a metric and an $S$-metric was investigated in various studies and some examples of an $S$-metric which is not generated by any metric were given (see 4, 5, 11 for more details).

Recently, the fixed circle problem (resp. fixed disc problem) raised by Özgür and Taş (see 12, 18] and the references therein) has been studied as an geometric

2020 Mathematics Subject Classification. Primary 54H25; Secondary 47H09, 47H10.

Keywords and phrases. $S$-metric space, fixed circle, $S$-Pata type $x_{0}$-mapping, $S$-Pata Zamfirescu type $x_{0}$-mapping.

\ihaltas@balikesir.edu.tr; nyozgur@yahoo.com-Corresponding author

(D) 0000-0002-4535-4019; 0000-0002-8152-1830.

(C) 2020 Ankara University

Communications Faculty of Sciences University of Ankara-Series A1 Mathematics and Statistics 
approach to the fixed-point theory on metric spaces and some generalized metric spaces (for example, $S$-metric spaces) (see $8,9,13,14,23,24$ ).

Now we recall the notions of a circle and a disc on an $S$-metric space given in [13 20], respectively.

Let $(X, \mathcal{S})$ be an $S$-metric space and $T: X \rightarrow X$ be a self-mapping. A circle $C_{x_{0}, r}^{S}$ and a disc $D_{x_{0}, r}^{S}$ are defined as follows:

$$
C_{x_{0}, r}^{S}=\left\{x \in X: \mathcal{S}\left(x, x, x_{0}\right)=r\right\}
$$

and

where $r \in[0, \infty)$.

$$
D_{x_{0}, r}^{S}=\left\{x \in X: \mathcal{S}\left(x, x, x_{0}\right) \leq r\right\},
$$

If $T x=x$ for all $x \in C_{x_{0}, r}^{S}$ (resp. $x \in D_{x_{0}, r}^{S}$ ), then the circle $C_{x_{0}, r}^{S}$ (resp. the disc $D_{x_{0}, r}^{S}$ ) is called as the fixed circle (resp. the fixed disc) of $T$.

A recent solution to the fixed-circle problem was given using the notion of $S$ Zamfirescu type $x_{0}$-mapping on $S$-metric spaces as follows:

Definition 2. [16] Let $(X, \mathcal{S})$ be an $S$-metric space and $T: X \rightarrow X$ be a selfmapping. Then $\bar{T}$ is called an $S$-Zamfirescu type $x_{0}$-mapping if there exist $x_{0} \in X$ and $a, b \in[0,1)$ such that

$$
\begin{aligned}
& \mathcal{S}(T x, T x, x)>0 \Longrightarrow \\
& \mathcal{S}(T x, T x, x) \leq \max \left\{a \mathcal{S}\left(x, x, x_{0}\right), \frac{b}{2}\left[\mathcal{S}\left(T x_{0}, T x_{0}, x\right)+\mathcal{S}\left(T x, T x, x_{0}\right)\right]\right\},
\end{aligned}
$$

for all $x \in X$.

Let the number $\delta$ be defined as

$$
\delta=\inf \{\mathcal{S}(T x, T x, x): T x \neq x, x \in X\} .
$$

Theorem 3. [16] Let $(X, \mathcal{S})$ be an $S$-metric space, $T: X \rightarrow X$ be a self-mapping and $\delta$ be the real number defined in (1). If the following conditions hold:

(i) $T$ is an $S$-Zamfirescu type $x_{0}$-mapping with $x_{0} \in X$,

(ii) $\mathcal{S}\left(T x, T x, x_{0}\right) \leq \delta$ for each $x \in C_{x_{0}, \delta}^{S}$,

then $C_{x_{0}, \delta}^{S}$ is a fixed circle of $T$, that is, $C_{x_{0}, \delta}^{S} \subset$ Fix $(T)$.

In this paper, we give new solutions to the fixed circle (resp. fixed disc) problem on $S$-metric spaces. In Section 2 we prove some fixed circle and fixed disc results using different approaches. In Section 3, we give some illustrative examples of our obtained results and deduce some important remarks. In Section 4 we summarize our study and recommend some future works.

\section{Main Results}

In this section, we inspire the methods given in $2,6,19,26$ and use the number defined in (1) to obtain new fixed circle (resp. fixed disc) results on $S$-metric spaces. In [19], Pata proved a fixed point theorem to generalize the well-known Banach's 
contraction principle on a metric space. However, Berinde showed that the main result in 19 does not hold at least in two extremal cases for the used parameter $\varepsilon$. The corrected version of this theorem was given with some necessary examples in [2]. In our results, we will not use the Picard iteration. Hence, our main results hold for all the parameters $\mu \in[0,1]$ and this situation will be supported by some illustrative examples given in the next section.

Let $\Theta$ denotes the class of all increasing functions $\psi:[0,1] \rightarrow[0, \infty)$ with $\psi(0)=0$.

Definition 4. Let $(X, \mathcal{S})$ be an $S$-metric space, $T: X \rightarrow X$ be a self-mapping, $\alpha \geq 0, \beta \geq 1$ and $\gamma \in[0, \beta]$ be any constants. Then $T$ is called an $S$-Pata type $x_{0}$-mapping if there exist $x_{0} \in X$ and $\psi \in \Theta$ such that

$\mathcal{S}(T x, T x, x)>0 \Longrightarrow \mathcal{S}(T x, T x, x) \leq \frac{1-\mu}{2}\|x\|_{s}+\alpha \mu^{\beta} \psi(\mu)\left[1+\|x\|_{s}+\|T x\|_{s}\right]^{\gamma}$, for all $x \in X$ and each $\mu \in[0,1]$, where $\|x\|_{s}=\mathcal{S}\left(x, x, x_{0}\right)$.

Notice that $\left\|x_{0}\right\|_{s}=\mathcal{S}\left(x_{0}, x_{0}, x_{0}\right)=0$. Let us consider the inequality given in the notion of $S$-Pata type $x_{0}$-mapping under the cases $\mu=0$ and $\mu=1$, respectively. For $\mu=0$, we have

$$
\mathcal{S}(T x, T x, x)>0 \Longrightarrow \mathcal{S}(T x, T x, x) \leq \frac{1}{2}\|x\|_{s}=\frac{\mathcal{S}\left(x, x, x_{0}\right)}{2}
$$

and also for $\mu=1$, we get

$$
\begin{aligned}
\mathcal{S}(T x, T x, x) & >0 \Longrightarrow \mathcal{S}(T x, T x, x) \leq \alpha \psi(1)\left[1+\|x\|_{s}+\|T x\|_{s}\right]^{\gamma} \\
& =L\left[1+\|x\|_{s}+\|T x\|_{s}\right]^{\gamma} \\
& =L\left[1+\mathcal{S}\left(x, x, x_{0}\right)+\mathcal{S}\left(T x, T x, x_{0}\right)\right]^{\gamma},
\end{aligned}
$$

where $L=\alpha \psi(1)>0$.

Theorem 5. Let $(X, \mathcal{S})$ be an $S$-metric space, $T: X \rightarrow X$ be an S-Pata type $x_{0}$-mapping with $x_{0} \in X$ and $\delta$ be the real number defined in $[1]$. Then $C_{x_{0}, \delta}^{S}$ is a fixed circle of $T$, that is, $C_{x_{0}, \delta}^{S} \subset F i x(T)$.

Proof. At first, we show that $x_{0}$ is a fixed point of $T$. On the contrary, assume that $T x_{0} \neq x_{0}$. Using the $S$-Pata type $x_{0}$-mapping property, we obtain

$$
\mathcal{S}\left(T x_{0}, T x_{0}, x_{0}\right) \leq \frac{1-\mu}{2}\left\|x_{0}\right\|_{s}+\alpha \mu^{\beta} \psi(\mu)\left[1+\left\|x_{0}\right\|_{s}+\left\|T x_{0}\right\|_{s}\right]^{\gamma} .
$$

For $\mu=0$, by inequality (2), we find

$$
\mathcal{S}\left(T x_{0}, T x_{0}, x_{0}\right) \leq 0
$$

this is a contradiction. So, the assumption is false. This shows that $T x_{0}=x_{0}$ and hence $\left\|T x_{0}\right\|_{s}=\left\|x_{0}\right\|_{s}=0$.

Let $\delta=0$. Then we have $C_{x_{0}, \delta}^{S}=\left\{x_{0}\right\}$. Clearly, $C_{x_{0}, \delta}^{S}$ is a fixed circle of $T$, that is, $C_{x_{0}, \delta}^{S} \subset F i x(T)$. 
Let $\delta>0$ and $x \in C_{x_{0}, \delta}^{S}$ be any point such that $T x \neq x$. Using the $S$-Pata type $x_{0}$-mapping hypothesis, we obtain

$$
\mathcal{S}(T x, T x, x) \leq \frac{1-\mu}{2}\|x\|_{s}+\alpha \mu^{\beta} \psi(\mu)\left[1+\|x\|_{s}+\|T x\|_{s}\right]^{\gamma} .
$$

For $\mu=0$, by inequality (3), we get

$$
\mathcal{S}(T x, T x, x) \leq \frac{1}{2}\|x\|_{s}=\frac{\mathcal{S}\left(x, x, x_{0}\right)}{2}=\frac{\delta}{2},
$$

a contradiction with the definition of $\delta$. Hence it should be $T x=x$. Consequently, $T$ fixes the circle $C_{x_{0}, \delta}^{S}$ and so $C_{x_{0}, \delta}^{S} \subset F i x(T)$.

Corollary 6. Let $(X, \mathcal{S})$ be an $S$-metric space, $T: X \rightarrow X$ be an $S$-Pata type $x_{0}$-mapping with $x_{0} \in X$ and $\delta$ be the real number defined in (1). Then $T$ fixes whole of the disc $D_{x_{0}, \delta}^{S}$, that is, $D_{x_{0}, \delta}^{S} \subset F i x(T)$.

Proof. By the similar arguments used in the proof of Theorem 5 , the proof follows easily.

We define another contractive condition to obtain a new fixed-circle result.

Definition 7. Let $(X, \mathcal{S})$ be an $S$-metric space, $T: X \rightarrow X$ be a self-mapping, $\alpha \geq 0, \beta \geq 1$ and $\gamma \in[0, \beta]$ be any constants. If there exist $x_{0} \in X$ and $\psi \in \Theta$ such that

$$
\begin{aligned}
\mathcal{S}(T x, T x, x)>\quad & \Longrightarrow \mathcal{S}(T x, T x, x) \leq \frac{1-\mu}{2} M_{S}\left(x, x_{0}\right) \\
& +\alpha \mu^{\beta} \psi(\mu)\left[1+\|x\|_{s}+\|T x\|_{s}+\left\|T x_{0}\right\|_{s}\right]^{\gamma},
\end{aligned}
$$

for all $x \in X$ and each $\mu \in[0,1]$, where $\|x\|_{s}=\mathcal{S}\left(x, x, x_{0}\right)$ and

$$
\begin{aligned}
& M_{S}(x, y) \\
= & \max \left\{\mathcal{S}(x, x, y), \frac{\mathcal{S}(T x, T x, x)+\mathcal{S}(T y, T y, y)}{2}, \frac{\mathcal{S}(T y, T y, x)+\mathcal{S}(T x, T x, y)}{2}\right\},
\end{aligned}
$$

then $T$ is called an $S$-Pata Zamfirescu type $x_{0}$-mapping with respect to $\psi \in \Theta$.

In the above definition, we consider the extremal cases $\mu=0$ and $\mu=1$, respectively. For $\mu=0$, we have

$$
\mathcal{S}(T x, T x, x)>0 \Longrightarrow \mathcal{S}(T x, T x, x) \leq \frac{1}{2} M_{S}\left(x, x_{0}\right)
$$

and also for $\mu=1$, we get

$$
\begin{aligned}
\mathcal{S}(T x, T x, x) & >0 \Longrightarrow \mathcal{S}(T x, T x, x) \leq \alpha \psi(1)\left[1+\|x\|_{s}+\|T x\|_{s}+\left\|T x_{0}\right\|_{s}\right]^{\gamma} \\
& =L\left[1+\|x\|_{s}+\|T x\|_{s}+\left\|T x_{0}\right\|_{s}\right]^{\gamma},
\end{aligned}
$$

where $L=\alpha \psi(1)>0$.

Now we investigate the relationship between the notions of an $S$-Zamfirescu type $x_{0}$-mapping and an $S$-Pata Zamfirescu type $x_{0}$-mapping. 
Let $\xi=\max \{a, b\}$ in Definition 2 and let us consider Bernoulli's inequality $1+p t \leq(1+t)^{p}, p \geq 1, t \in[-1, \infty)$. Then we get

$$
\begin{aligned}
& \mathcal{S}(T x, T x, x)>0 \Longrightarrow \\
& \mathcal{S}(T x, T x, x) \leq \max \left\{a \mathcal{S}\left(x, x, x_{0}\right), \frac{b}{2}\left[\mathcal{S}\left(T x_{0}, T x_{0}, x\right)+\mathcal{S}\left(T x, T x, x_{0}\right)\right]\right\} \\
& \leq \xi \max \left\{\mathcal{S}\left(x, x, x_{0}\right), \frac{\mathcal{S}\left(T x_{0}, T x_{0}, x\right)+\mathcal{S}\left(T x, T x, x_{0}\right)}{2}\right\} \\
& \leq \xi \max \left\{\mathcal{S}\left(x, x, x_{0}\right), \frac{\mathcal{S}(T x, T x, x)+\mathcal{S}\left(T x_{0}, T x_{0}, x_{0}\right)}{2}, \frac{\mathcal{S}\left(T x_{0}, T x_{0}, x\right)+\mathcal{S}\left(T x, T x, x_{0}\right)}{2}\right\} \\
& \leq \frac{1-\mu}{2} \max \left\{\mathcal{S}\left(x, x, x_{0}\right), \frac{\mathcal{S}(T x, T x, x)+\mathcal{S}\left(T x_{0}, T x_{0}, x_{0}\right)}{2}, \frac{\mathcal{S}\left(T x_{0}, T x_{0}, x\right)+\mathcal{S}\left(T x, T x, x_{0}\right)}{2}\right\} \\
& +\left(\xi+\frac{\mu-1}{2}\right)\left[1+\max \left\{\|x\|_{s}, \frac{\|x\|_{s}+\|T x\|_{s}+\left\|T x_{0}\right\|_{s}}{2}\right\}\right] \\
& \leq \frac{1-\mu}{2} M_{S}\left(x, x_{0}\right)+\xi\left(1+\frac{\mu-1}{\xi}\right)\left[1+\|x\|_{s}+\|T x\|_{s}+\left\|T x_{0}\right\|_{s}\right] \\
& \leq \frac{1-\mu}{2} M_{S}\left(x, x_{0}\right)+\xi \mu^{\frac{1}{\xi}}\left[1+\|x\|_{s}+\left\|x_{0}\right\|_{s}+\|T x\|_{s}+\left\|T x_{0}\right\|_{s}\right] \\
& \leq \frac{1-\mu}{2} M_{S}\left(x, x_{0}\right)+\xi \mu \mu^{\frac{1-\xi}{\xi}}\left[1+\|x\|_{s}+\left\|x_{0}\right\|_{s}+\|T x\|_{s}+\left\|T x_{0}\right\|_{s}\right] .
\end{aligned}
$$

Hence we get that an $S$-Zamfirescu type $x_{0}$-mapping is a special case of an $S$-Pata Zamfirescu type $x_{0}$-mapping with $\alpha=\xi, \psi(x)=x^{\frac{1-\xi}{\xi}}$ and $\beta=\gamma=1$.

Now we prove the following fixed circle theorem.

Theorem 8. Let $(X, \mathcal{S})$ be an $S$-metric space, $T: X \rightarrow X$ be a self-mapping and $\delta$ be the real number defined in (1). If the following conditions hold:

(i) $T$ is an $S$-Pata Zamfirescu type $x_{0}$-mapping with respect to $\psi \in \Theta$ for $x_{0} \in X$,

(ii) $\mathcal{S}\left(T x, T x, x_{0}\right) \leq \delta$ for each $x \in C_{x_{0}, \delta}^{S}$,

then $C_{x_{0}, \delta}^{S}$ is a fixed circle of $T$, that is, $C_{x_{0}, \delta}^{S} \subset F i x(T)$.

Proof. Using the condition $(i)$, we can easily obtain that $T x_{0}=x_{0}$ and hence $\left\|T x_{0}\right\|_{s}=\left\|x_{0}\right\|_{s}=0$. Let $\delta=0$. Then we have $C_{x_{0}, \delta}^{S}=\left\{x_{0}\right\}$. Clearly, $C_{x_{0}, \delta}^{S}$ is a fixed circle of $T$, that is, $C_{x_{0}, \delta}^{S} \subset F i x(T)$.

Let $\delta>0$ and $x \in C_{x_{0}, \delta}^{S}$ be any point such that $T x \neq x$. Using the conditions (i) and (ii), we obtain

$$
\begin{aligned}
\mathcal{S}(T x, T x, x) \leq & \frac{1-\mu}{2} M_{S}\left(x, x_{0}\right)+\alpha \mu^{\beta} \psi(\mu)\left[1+\|x\|_{s}+\|T x\|_{s}+\left\|T x_{0}\right\|_{s}\right]^{\gamma} \\
\leq & \frac{1-\mu}{2} \max \left\{\delta, \frac{\mathcal{S}(T x, T x, x)}{2}\right\} \\
& +\alpha \mu^{\beta} \psi(\mu)\left[1+\|x\|_{s}+\|T x\|_{s}\right]^{\gamma} .
\end{aligned}
$$

For $\mu=0$, using the inequality (4), we get

$$
\mathcal{S}(T x, T x, x) \leq \frac{1}{2} \max \left\{\delta, \frac{\mathcal{S}(T x, T x, x)}{2}\right\},
$$

a contradiction with the definition of $\delta$. Consequently, it should be $T x=x$ whence $T$ fixes the circle $C_{x_{0}, \delta}^{S}$ and so $C_{x_{0}, \delta}^{S} \subset F i x(T)$. 
Corollary 9. Let $(X, \mathcal{S})$ be an $S$-metric space, $T: X \rightarrow X$ be a self-mapping and $\delta$ be the real number defined in (1). If the following conditions hold:

(i) $T$ is an $S$-Pata Zamfirescu type $x_{0}$-mapping with respect to $\psi \in \Theta$ for $x_{0} \in X$,

(ii) $\mathcal{S}\left(T x, T x, x_{0}\right) \leq \delta$ for each $x \in D_{x_{0}, \delta}^{S}$

then $T$ fixes whole of the disc $D_{x_{0}, \delta}^{S}$, that is, $D_{x_{0}, \delta}^{S} \subset F i x(T)$.

Proof. By the similar arguments used in the proof of Theorem 8 , the proof follows easily.

Remark 10. If a self-mapping $T$ satisfies the conditions of Theorem 8, then we have $\left\|T x_{0}\right\|_{s}=\left\|x_{0}\right\|_{s}=0$. Therefore, Theorem 8 coincides with Theorem 5 in the case where $M_{S}\left(x, x_{0}\right)=\|x\|_{s}$ for all $x \in X$. On the other hand, if $T$ satisfies the conditions of Theorem 5 then clearly, $T$ satisfies the conditions of Theorem 8 since $M_{S}\left(x, x_{0}\right) \geq\|x\|_{s}$.

\section{Illustrative Examples and Some Remarks}

In this section, we give some examples to show the validity of our results obtained in the previous section.

Example 11. Let $X=\mathbb{R}$ be the $S$-metric space with the $S$-metric defined by

$$
\mathcal{S}(x, y, z)=|x-z|+|x+z-2 y|,
$$

for all $x, y, z \in \mathbb{R}\left[11\right.$. Let us define the self-mapping $T_{1}: \mathbb{R} \rightarrow \mathbb{R}$ as

$$
T_{1} x=\left\{\begin{array}{cc}
x & , \quad \\
x+\frac{1}{2} & , \quad x \in(-\infty,-2) \cup(2, \infty)
\end{array},\right.
$$

for all $x \in \mathbb{R}$. Then $T_{1}$ is both an $S$-Pata type $x_{0}$-mapping and an S-Pata Zamfirescu type $x_{0}$-mapping with $x_{0}=0, \alpha=\beta=\gamma=1$ and

$$
\psi(x)=\left\{\begin{array}{lc}
0 & , \quad x=0 \\
\frac{1}{2} & , \quad x \in(0,1]
\end{array} .\right.
$$

Also we have $\delta=1$. Consequently, by Theorem 5 and Theorem 8 (resp. Corollary 6 and Corollary 9), $T_{1}$ fixes the circle $C_{0,1}^{S}=\left\{-\frac{1}{2}, \frac{1}{2}\right\}$ (resp. the disc $D_{0,1}^{S}=\left[-\frac{1}{2}, \frac{1}{2}\right]$ ).

Example 12. Let $X=\mathbb{R}$ be the $S$-metric space with the $S$-metric considered in Example 11, Let us define the self-mapping $T_{2}: \mathbb{R} \rightarrow \mathbb{R}$ as

$$
T_{2} x=\left\{\begin{array}{cc}
x & , \quad x \in[-4, \infty) \\
x+1 & , \quad x \in(-\infty,-4)
\end{array},\right.
$$

for all $x \in \mathbb{R}$. Then $T_{2}$ is both an $S$-Pata type $x_{0}$-mapping and an S-Pata Zamfirescu type $x_{0}$-mapping with $x_{0}=0\left(\right.$ or $\left.x_{0}=3\right), \alpha=\beta=\gamma=1$ and

$$
\psi(x)=\left\{\begin{array}{lc}
0 & , \quad x=0 \\
\frac{1}{2} & , \quad x \in(0,1]
\end{array} .\right.
$$

Also we obtain $\delta=2$. Consequently, $T_{2}$ fixes the circles $C_{0,2}^{S}$ and $C_{3,2}^{S}$ (resp. the discs $D_{0,2}^{S}$ and $\left.D_{3,2}^{S}\right)$. 
Example 13. Let $X=\mathbb{R}$ be the $S$-metric space with the $S$-metric considered in Example 11. Let us define the self-mapping $T_{3}: \mathbb{R} \rightarrow \mathbb{R}$ as

$$
T_{3} x=\left\{\begin{array}{cc}
x & , \quad x \in[-2,2] \\
0 & , \quad x \in(-\infty,-2) \cup(2, \infty)
\end{array},\right.
$$

for all $x \in \mathbb{R}$. Then $T_{3}$ is not an $S$-Pata type $x_{0}$-mapping and an $S$-Pata Zamfirescu type $x_{0}$-mapping with $x_{0}=0$. But $T_{3}$ fixes the circle $C_{0,4}^{S}=\{-2,2\}$ and the disc $D_{0,4}^{S}=[-2,2]$.

Now, we give an example of a self-mapping that satisfies the conditions of Theorem 8 but does not satisfy the conditions of Theorem 5 .

Example 14. Let $X=\mathbb{R}$ be the $S$-metric space with the usual $S$-metric defined by

$$
\mathcal{S}(x, y, z)=|x-z|+|y-z|,
$$

for all $x, y, z \in X$ [21]. Now, we define the self-mapping $T_{4}: X \rightarrow X$ by

$$
T_{4} x=\left\{\begin{array}{cc}
\frac{5}{3} x & , \quad|x|=1 \\
x & , \quad|x| \neq 1
\end{array}\right.
$$

We have

$$
\begin{aligned}
\delta & =\inf \left\{\mathcal{S}\left(T_{4} x, T_{4} x, x\right):|x|=1\right\} \\
& =\inf \left\{2\left|T_{4} x-x\right|:|x|=1\right\} \\
& =\inf \left\{2\left|\frac{5}{3} x-x\right|:|x|=1\right\} \\
& =\inf \left\{\frac{4}{3}|x|:|x|=1\right\}=\frac{4}{3} .
\end{aligned}
$$

Then, it is easy to verify that $T_{4}$ is not an $S$-Pata type $x_{0}$-mapping for the point $x_{0}=0$ independent from the choice of the parameters $\alpha, \beta, \gamma$ and the function $\psi$. But, if we choose $\alpha=\beta=\gamma=1$ then $T_{4}$ is an S-Pata Zamfirescu type $x_{0}$-mapping for the point $x_{0}=0$ with the function

$$
\psi(x)=\left\{\begin{array}{lcc}
0 & , \quad x=0 \\
\frac{1}{4} & , \quad x \in(0,1]
\end{array} .\right.
$$

Clearly, $T_{4}$ fixes the circle

$$
\begin{aligned}
C_{0, \frac{4}{3}}^{S} & =\left\{x: 2|x-0|=\frac{4}{3}\right\} \\
& =\left\{x:|x|=\frac{2}{3}\right\} \\
& =\left\{-\frac{2}{3}, \frac{2}{3}\right\}
\end{aligned}
$$

and the disc $D_{0, \frac{4}{3}}^{S}=\left\{x:|x| \leq \frac{2}{3}\right\}$. 
The following remarks can be deduced from the obtained results and the given examples.

Remark 15. (i) The point $x_{0}$ satisfying the conditions of an S-Pata type $x_{0}$ mapping and an S-Pata Zamfirescu type $x_{0}$-mapping is always a fixed point of the self-mapping T. Moreover, the choice of $x_{0}$ is independent from the number $\delta$ (see Example 11 and Example 12). Also the number of $x_{0}$ can be more than one (see Example 12).

(ii) The converse statements of Theorem 5, Corollary 6. Theorem 8 and Corollary 9 are not always true (see Example 13). That is, a self-mapping having a fixed circle (resp. fixed disc) need not to be an S-Pata type $x_{0}$-mapping or an S-Pata Zamfirescu type $x_{0}$-mapping with $x_{0}$ where the point $x_{0}$ is the center of the fixed circle (resp. fixed disc).

\section{Conclusion}

In this paper, we have presented some new solutions to the fixed circle problem on $S$-metric spaces. To do this, we have inspired by the Pata and Zamfirescu type methods. We have proved two main fixed circle theorems and some related results. Also, we have given necessary illustrative examples supporting our obtained results. On the other hand, there are many generalized metric spaces in the literature (for example, see [3, 25] and the references therein). Hence, the fixed circle (resp. fixed disc) problem can be studied on these generalized metric spaces using similar approaches as a future work.

On the other hand, a related problem is the best proximity point problem since the best proximity point theorems investigate an optimal solution of the minimization problem $\{d(x, T x): x \in A\}$ for a mapping $T: A \rightarrow B$ where $A$ and $B$ are two non-empty subsets of a metric space (see 1$]$ and the references therein). In [6], the existence of best proximity point was investigated using the Pata type proximal mappings. In [17, the notion of a best proximity circle is introduced and some proximal contractions for a non-self-mapping are determined. In this context, a related future work is the investigation of the existence of a best proximity circle via the notions of $p$-proximal contraction and $p$-proximal contractive mapping defined in 22 .

Acknowledgement. Both authors are supported by the Scientific Research Projects Unit of Balikesir University under the project number BAP 2018 / 021.

\section{REFERENCES}

[1] Altun, İ., Aslantaş, M., Şahin, H., Best proximity point results for p-proximal contractions, Acta Math. Hungar., (2020). https://doi.org/10.1007/s10474-020-01036-3.

[2] Berinde, V., Comments on some fixed point theorems in metric spaces, Creat. Math. Inform., 27 (1) (2018), 15-20.

[3] Dosenovic, T., Radenovic, S. and Sedghi, S., Generalized metric spaces: survey, TWMS J. Pure Appl. Math., 9 (1) (2018), 3-17.

[4] Gupta, A., Cyclic contraction on S-metric space, Int. J. Anal. Appl., 3 (2) (2013), 119-130. 
[5] Hieu, N. T., Ly, N. T., Dung, N. V., A generalization of Ciric quasi-contractions for maps on S-metric spaces, Thai J. Math., 13 (2) (2015), 369-380.

[6] Jacob, G. K., Khan, M. S., Park, C., Yun, S., On generalized Pata type contractions, Mathematics, 6 (2018), 25.

[7] Mlaiki, N., $\alpha$ - $\psi$-contractive mapping on $S$-metric space, Math. Sci. Lett., 4 (1) (2015), 9-12.

[8] Mlaiki, N., Çelik, U., Taş, N., Özgür, N. Y., Mukheimer, A., Wardowski type contractions and the fixed-circle problem on $S$-metric spaces, J. Math., (2018), Article ID 9127486.

[9] Mlaiki, N., Özgür, N. Y., Taş, N., New fixed-point theorems on an $S$-metric space via simulation functions, Mathematics, 7(7) (2019), 583.

[10] Özgür, N. Y., Taş, N., Some fixed point theorems on S-metric spaces, Mat. Vesnik, 69 (1) (2017), 39-52.

[11] Özgür, N. Y., Taş, N., Some new contractive mappings on $S$-metric spaces and their relationships with the mapping (S25), Math. Sci. (Springer), 11 (1) (2017), 7-16.

[12] Özgür, N. Y., Taş, N., Some fixed-circle theorems on metric spaces, Bull. Malays. Math. Sci. Soc., 42 (4) (2019), 1433-1449.

[13] Özgür, N. Y., Taş, N., Fixed-circle problem on $S$-metric spaces with a geometric viewpoint, Facta Universitatis. Series: Mathematics and Informatics, 34 (3) (2019), 459-472.

[14] Özgür, N. Y., Taş, N., Çelik, U., New fixed-circle results on S-metric spaces, Bull. Math. Anal. Appl., 9 (2) (2017), 10-23.

[15] Özgür, N. Y., Taş, N., The Picard theorem on S-metric space, Acta Math. Sci., 38B (4) (2018), 1245-1258.

[16] Özgür, N. Y., Taş, N., A new solution to the Rhoades' open problem with an application, arXiv preprint. arXiv:1910.12304

[17] Özgür, N. Y., Taş, N., Pata Zamfirescu type fixed-disc results with a proximal application, arXiv preprint. arXiv:1910.12302

[18] Özgür, N. Y., Fixed-disc results via simulation functions, Turkish J. Math., 43 (6) (2019), 2794-2805.

[19] Pata, V., A fixed point theorem in metric spaces, J. Fixed Point Theory Appl., 10 (2011), 299-305.

[20] Sedghi, S., Shobe, N., Aliouche, A., A generalization of fixed point theorems in $S$-metric spaces, Mat. Vesnik, 64 (3) (2012), 258-266.

[21] Sedghi, S., Dung, N. V., Fixed point theorems on S-metric spaces, Mat. Vesnik, 66 (1) (2014), 113-124.

[22] Şahin, H., Aslantas, M., Altun, İ., Feng-Liu type approach to best proximity point results for multivalued mappings. J. Fixed Point Theory Appl. 22 (2020), 11.

[23] Taş, N., Suzuki-Berinde type fixed-point and fixed-circle results on $S$-metric spaces, J. Linear Topol. Algebra, 7 (3) (2018), 233-244.

[24] Taş, N., Various types of fixed-point theorems on S-metric spaces, J. BAUN Inst. Sci. Technol., 20 (2) (2018), 211-223.

[25] Taş, N., Özgür, N. Y., New generalized fixed point results on $S_{b}$-metric spaces, arXiv preprint. arXiv: 1703.01868

[26] Zamfirescu, T., Fixed point theorems in metric spaces, Arch. Math., 23 (1972), 292-298. 